\title{
Detecting joint inflammation by an LED-based photoacoustic imaging system: a feasibility study (Erratum)
}

\author{
Janggun Jo, ${ }^{\mathrm{a}, \uparrow}$ Guan Xu ${ }^{\mathrm{b},{ }^{\dagger} \dagger}$ Yunhao Zhu, ${ }^{\mathrm{a}}$ Mary Burton, ${ }^{\mathrm{b}}$ Jeffrey Sarazin, ${ }^{\mathrm{c}}$ \\ Elena Schiopu, ${ }^{\mathrm{c}}$ Girish Gandikota, ${ }^{\mathrm{b}}$ and Xueding Wang ${ }^{\mathrm{a}, \mathrm{b}}$ \\ ${ }^{a}$ University of Michigan, Department of Biomedical Engineering, Ann Arbor, Michigan, \\ United States \\ ${ }^{b}$ University of Michigan Medical School, Department of Radiology, Ann Arbor, Michigan, \\ United States \\ 'University of Michigan Medical School, Division of Rheumatology, \\ Department of Internal Medicine, Ann Arbor, Michigan, United States
}

[DOI: 10.1117/1.JBO.26.5.059802]

This article [J. Biomed. Opt. 23(11), 110501 (2018) doi: 10.1117/1.JBO.23.11.110501 was originally published on 29 November 2018 with an error in the name of the first author. Janggun Jo was misspelled as "Junggun Jo." The error was corrected on 7 May 2021.

Address all correspondence to: Girish Gandikota, E-mail: ggirish@med.umich.edu; Xueding Wang, E-mail: xdwang@umich.edu 